Research Paper

\title{
HPV E7 inhibits cell pyroptosis by promoting TRIM21- mediated degradation and ubiquitination of the IFI16 inflammasome
}

\author{
Yinjing Song1 ${ }^{*}$, Xia $\mathrm{Wu}^{1 *}$, Yaohan $\mathrm{Xu}^{1 *}$, Jiang Zhu ${ }^{1}$, Jiaying $\mathrm{Li}^{2}$, Ziqi Zou ${ }^{2}$, Luxia Chen ${ }^{1}$, Boya Zhang1, \\ Chunting Hua ${ }^{1}$, Han Rui ${ }^{1}$, Qiaoli Zheng ${ }^{1}$, Qiang Zhou ${ }^{1 凶}$, Qingqing Wang ${ }^{2 \bowtie}$ and Hao Cheng ${ }^{1 凶}$ \\ 1. Department of Dermatology and Venereology, Sir Run Run Shaw Hospital, Zhejiang University School of Medicine, Hangzhou 310016, Zhejiang, PR China. \\ 2. Institute of Immunology, Zhejiang University School of Medicine, Hangzhou 310058, China. \\ *These authors contributed equally to this work. \\ $\triangle$ Corresponding author: Hao Cheng, E-mail: chenghao1@zju.edu.cn, or Qiang Zhou, E-mail: zhouqiangdr@126.com; Department of Dermatology and \\ Venereology, Sir Run Run Shaw Hospital Zhejiang University School of Medicine, Zhejiang Province, China, 310016. Tel: 86-571-86006972; Fax: 86-571-86044817; \\ Qingqing Wang, E-mail: wqq@zju.edu.cn, Institute of Immunology, Zhejiang University School of Medicine, Zhejiang Province, China, 310058. Tel: \\ +86-571-88208284; Fax: +86-571-88208285.
}

(c) The author(s). This is an open access article distributed under the terms of the Creative Commons Attribution License (https://creativecommons.org/licenses/by/4.0/). See http://ivyspring.com/terms for full terms and conditions.

Received: 2020.06.29; Accepted: 2020.08.30; Published: 2020.09.13

\begin{abstract}
Human papillomavirus (HPV) is a DNA virus that causes sexually transmitted infections. The HPV oncoprotein E7 plays a critical role in the regulation of host immunity to promote the immune escape of HPV and the occurrence of cervical cancer or genital warts. Pyroptosis, a highly inflammatory form of programmed cell death, can be induced by inflammasomes and acts as a defense against pathogenic infection. However, whether HPV E7 can regulate cell pyroptosis to evade immune surveillance has not been determined. In this study, we found that HPV E7 could inhibit cell pyroptosis induced by transfection with dsDNA. The activation of the inflammasome, and the production of IL-18 and IL-1 $\beta$ were also restrained by HPV E7. Mass spectrometry and immunoprecipitation showed that HPV E7 interacted with IFI16 and TRIM21. We also discovered that HPV E7 recruited the E3 ligase TRIM21 to ubiquitinate and degrade the IFI16 inflammasome, leading to the inhibition of cell pyroptosis and self-escape from immune surveillance. Thus, our study reveals an important immune escape mechanism in HPV infection and may provide targets for the development of a novel immunotherapeutic strategy to effectively restore antiviral immunity.
\end{abstract}

Key words: Human papillomavirus E7; pyroptosis; ubiquitination; inflammasome

\section{Introduction}

Human papillomavirus (HPV) is a small doublestranded DNA virus that infects skin or mucosal cells and causes the development of genital warts or cancers [1]. Depending on the oncogenic potential cancers, HPV types are classified into low-risk types such as HPV-6 and HPV-11 which are found mainly in genital warts, and high-risk types, such as HPV-16 and HPV-18 which are frequently associated with cervical cancer [2]. The induction of immune evasion by HPV is one of the most important mechanisms in persistent HPV infection and is responsible for HPVassociated disease progression [3]. Accumulating evidence shows that the both low-risk and high-risk HPV E7 plays an important role in immune evasion through various mechanisms [4-6]. For example, both low-risk and high-risk HPV E7 proteins inhibit the expression of IRF1 by recruiting HDAC to promoters that contain IRF1 response elements [7]. The production of IL-18 binding protein (IL-18BP) which can prevent the deleterious effects of excessive IL-18 secretion is increased in primary human keratinocytes expressing either high-or low-risk HPVE7 [8]. HPV E7 also restrain the expression of interferons (IFNs), proinflammatory cytokines, chemokines, TLRs and 
MHC-I by deregulating host DNA methylation and histone modification [9-12]. Moreover, HPV E7 can locate in the cytoplasm to interrupt the cGAS-STING and IFN signaling pathways by interacting with STING, IRF9 or IRF1 [13-15]. Despite these findings, the break in host immune surveillance induced by HPV E7 is far from fully understood, which largely restricts the efficacy of immunotherapy against HPV.

Host cell death, including apoptosis, necroptosis, and pyroptosis, is critical for host defenses against infection and the clearing of intracellular pathogens $[16,17]$. Pyroptosis is a recently identified host cell death pathway that occurs most frequently upon infection with intracellular pathogens $[18,19]$. It has recently been reported that pyroptosis is mediated by gasdermin D (GSDMD), which is cleaved by the inflammatory caspase- 1 or caspase-11 and forms pores on membranes, resulting in the extracellular release of IL-1 $\beta$ and-18, cell swelling and pyrolysis $[20,21]$. Nucleotide-binding oligomerization domainlike receptors (NLRs) and absent in melanoma 2 (AIM2)-like receptors are able to form multimeric inflammasome complexes and activate inflammatory caspases which initiate cell pyroptosis during pathogen infection [22]. Interferon-gamma inducible protein 16 (IFI16) is a member of the PYHIN family, and in addition to being able to promote cGASSTING-dependent interferon- $\beta$ (IFN- $\beta$ ) production, IFI16 can also assemble the inflammasome and initiate pyroptosis [23, 24]. For example, IFI16 detects viral DNA and initiates the assembly of inflammasome in both the cytoplasm and nucleus upon infection with DNA viruses such as Kaposi's sarcoma-associated herpesvirus (KSHV), Epstein-Barr virus (EBV) or herpes simplex virus (HSV-1) [25-28]. Moreover, human immunodeficiency virus [29] DNA can be sensed by IFI16 and induce the activation of IFI16 inflammasome in the cytoplasm, which mediates the pyroptosis of $\mathrm{CD}^{+} \mathrm{T}$ cells during abortive HIV-1 infection [30]. Therefore, the IFI16 inflammasome plays an important role in the initiation of pyroptosis during viral infection. However, whether HPV infection or HPV oncoproteins affect IFI16 inflammasome-mediated pyroptosis is unclear.

Here, we found that HPV E7 could inhibit cell pyroptosis induced by the IFI16 inflammasome. Mass spectrometry and immunoprecipitation showed that HPV E7 interacted with IFI16 and TRIM21. Furthermore, we found that HPV E7 promoted the K33-linked ubiquitination and degradation of IFI16 mediated by the E3 ligase TRIM21. Our data demonstrated that HPV E7 inhibited cell pyroptosis by promoting TRIM21-mediated degradation and K33-linked ubiquitination of the IFI16 inflammasome, thereby facilitating HPV immune escape.

\section{Materials and Methods}

\section{Plasmids, antibodies, and reagents}

Recombinant vectors encoding human IFI16 (NM_001364867.2), TRIM21 (NM_003141.4), and HPV 18E7 (NC_001357.1) were generated by PCR-based amplification of HeLa cell cDNA, followed by subcloning into the pcDNA3.1 eukaryotic expression vector or pEGFP-C1 vector. Complementary DNA (cDNA) for HPV 11E7 (GenBank: CCC55776.1) was amplified from condyloma acuminate tissues, and HPV 18E7 cDNAs were inserted into the pHAGEfEF1a-IRES-ZsGreen lentiviral vector with an $\mathrm{N}$-terminal Flag tag for lentiviral packaging. All constructs were confirmed by DNA sequencing. 18E7 siRNA was purchased from GenePharma.

Antibodies against GSDMD (96458s, 1:3000), caspase-1 (3866T, 1:3000), cleaved caspase-1 (4199T, 1:3000), and GAPDH (5174, 1:5000) were purchased from Cell Signaling Technology. Antibodies specific for IFI16 (sc-8023, 1:1000), TRIM21 (sc25351, 1:1000), ubiquitin (sc-471120, 1:1000), and GFP (sc-390394) were from Santa Cruz, Inc. Antibodies against Flag (M20008M), Myc (M20002M), mouse IgG (B30010M), and rabbit IgG (B30011S) were obtained from Abmart. Poly(dA:dT) (P0883), MG132 (M8699), CHX (C4859) and anti-FlagM2 magnetic beads (M8823) were purchased from Sigma-Aldrich. ELISA kits for human IL-18 and IL-1 $\beta$ were purchased from eBioscience.

\section{Cell culture and transfection}

The HeLa cervical cancer cell line (ATCC no. CCL2) and 293T cells (ATCC no. CRL-3216) were purchased from the American Type Culture Collection (ATCC, Manassas, VA, USA) and maintained in Dulbecco's modified Eagle's medium (DMEM) containing 10\% fetal bovine serum. For transient transfection, HeLa and 293T cells were transfected with plasmids using jetPRIME (PolyPlus-Transfection, 114-15). To develop stable expression cell lines, HaCaT or HeLa cells were transfected with lentivirus for $48 \mathrm{hr}$ and sorted by flow cytometry (BD Biosciences FACSAria II).

\section{Generation of knockout cell lines by CRISPR-Cas9}

pGE-4 (pU6gRNA1U6gRNA2Cas9puro) CRISPR-Cas9 plasmids were purchased from Gene Pharma. The target sequences used were ATGCTCAC AGGCTCCACGAA and CATGTTGGCTAGCTGTC GAT for TRIM21; and CTCAGTACCTTCACTATCAC and GGAATATGATAGTCTCCTAG for IFI16. To construct the knockout cell lines, HeLa cells were transfected with CRISPR-Cas9 and pEGFP-C1 vectors for $48 \mathrm{hr}$ and sorted into 96-well plates for single-cell 
sorting with a FACSAria II cell sorter (BD BioSciences). The candidate knockout clones were verified by western blotting.

\section{Mass spectrometry}

HaCaT cells stably expressing Flag-HPV 11E7 were transfected with poly(dA:dT) for $6 \mathrm{hrs}$ before collection. Cell lysates were immunoprecipitated with antibody against the Flag epitope tag, and mass spectrometry was used to identify Flag-HPV 11E7-interacting proteins with an Orbitrap Elite mass spectrometer (Thermo Fisher).

\section{Cytokine release assay}

IL-18 and IL- $\beta$ cytokines were detected with ELISA kits according to the manufacturer's protocols.

\section{Immunoprecipitation and immunoblot analysis}

For immunoprecipitation, cells were lysed in NP-40 lysis buffer (Beyotime, P0013F) with protease inhibitor 'cocktail' (Sigma, P8340) and then centrifuged for $15 \mathrm{~min}$ at $12,000 \times \mathrm{g}$. The supernatants were collected and incubated with protein A/G magnetic beads (Thermo Scientific Pierce, 88802) and a specific antibody at $4^{\circ} \mathrm{C}$ overnight. Immunoprecipitates were washed five times with IP wash buffer and eluted in SDS-PAGE loading buffer. For immunoblot analysis, whole-cell extracts were lysed with cell lysis buffer (Cell Signaling Technology, 9803) supplemented with a protease inhibitor 'cocktail' (Sigma, P8340). Protein concentrations in the extracts were measured by BCA assay (Pierce, 23227) according to the protocol. Equal amounts of extracts were separated by SDS-polyacrylamide gel electrophoresis and then transferred onto a polyvinylidene fluoride membrane (Millipore, IPVH00010). The polyvinylidene fluoride membrane was blocked with $5 \%$ dry nonfat milk in Tris-buffered saline (pH 7.4) containing $0.1 \%$ Tween 20 and probed with the appropriate antibody for immunoblot analysis.

\section{Flow cytometry}

HeLa cells were transfected with poly(dA:dT) for the indicated times, washed twice with cold PBS, and then resuspended in $1 \times$ binding buffer containing propidium iodide (BD Biosciences, 556547) for $15 \mathrm{~min}$ at RT $\left(25^{\circ} \mathrm{C}\right)$ in the dark. The stained cells were analyzed by flow cytometry (FACS).

\section{Cell viability and cell death}

For cell viability assays, HeLa or HaCaT cells were transfected with poly $(\mathrm{dA}: \mathrm{dT})$ for the indicated times, and their viability was detected with a CellTiter-Glo Luminescent Cell Viability Assay
(Promega, G7570). Cell death was measured with an LDH assay using a CytoTox 96 Non-Radioactive Cytotoxicity assay kit (Promega, G1780).

\section{Statistical Analysis}

All results are expressed as the mean \pm SEM of at least three independent experiments. Statistical significance was evaluated by Student's $t$-test. $P$ values less than 0.05 indicated statistical significance.

\section{Data availability}

All relevant data are available from the authors upon request.

\section{Results}

\section{HPV E7 inhibits pyroptosis induced by intracellular dsDNA}

To determine whether HPV E7 can regulate pyroptosis, we constructed and obtained HaCaT cells stably overexpressing low-risk HPV11 early gene E7 or HeLa cells stably overexpressing high-risk HPV18 oncoprotein E7 by infection with the lentivirus expressing Flag-HPV 11E7 or Flag-HPV 18E7, respectively (Fig. S1A). Flow cytometry analysis revealed that fewer HPV E7-overexpressing cells were propidium iodide-positive than control cells in response to transfection with poly $(\mathrm{dA}: \mathrm{dT})$ which is a repetitive synthetic double-stranded DNA sequence and recognized by multiple PRRs, such as cGAS, IFI16, DAI, AIM2, DDX41, and LRRFIP1 (Figure 1A-B). Compared to control cells, HPV E7overexpressing cells displayed higher cell viability, as detected by the CellTiter-Glo luminescent cell viability assay (Figure 1C). Furthermore, the lactate dehydrogenase release assay showed that HPV E7-overexpressing cells released less LDH than control cells in response to poly(dA:dT) (Figure 1D). We also found that overexpression of HPV E7 inhibited the cleavage of GSDMD in HaCaT or HeLa cells in response to transfection with poly (dA:dT) (Figure 1E). As GSDMD is cleaved by inflammatory caspase, we detected the activation of caspase- 1 and found that HPV E7-overexpressing cells displayed a lower level of activated caspase-1 than control cells (Fig. 1E). The levels of IL- $\beta$ and IL-18 were significantly decreased in HPV E7-overexpressing cells transfected with poly(dA:dT) (Figure 1F).Taken together, our results demonstrated that HPV E7 can inhibit cell pyroptosis induced by poly(dA:dT) transfection.

To further confirm the function of HPV E7 in regulating pyroptosis, we silenced $18 \mathrm{E} 7$ by shRNA in HeLa cells and transfected with poly(dA:dT). More 18E7 silenced HeLa cells were propidium iodidepositive than control HeLa cells in response to 
poly(dA:dT) (Figure S1B-1C). More LDH was released from 18E7 silenced HeLa cells than control cells in response to poly(dA:dT) (Figure S1D). However, the cell viability was significantly lower in 18E7 silenced HeLa cells than control HeLa cells after transfection with poly(dA:dT) (Figure S1E). Silence of 18E7 promoted the cleavage of GSDMD and activation of caspase- 1 in HeLa cells transfected with poly(dA:dT)
(Figure S1F). Furthermore, the production of IL-1 $\beta$ and IL-18 was markedly increased in 18E7 silenced HeLa cells transfected with poly(dA:dT) compared to control HeLa cells transfected with poly(dA:dT) (Figure S1G). Taken together, our results demonstrate that HPVE7 can inhibit cell pyroptosis induced by poly $(\mathrm{dA}: \mathrm{dT})$ transfection.
A

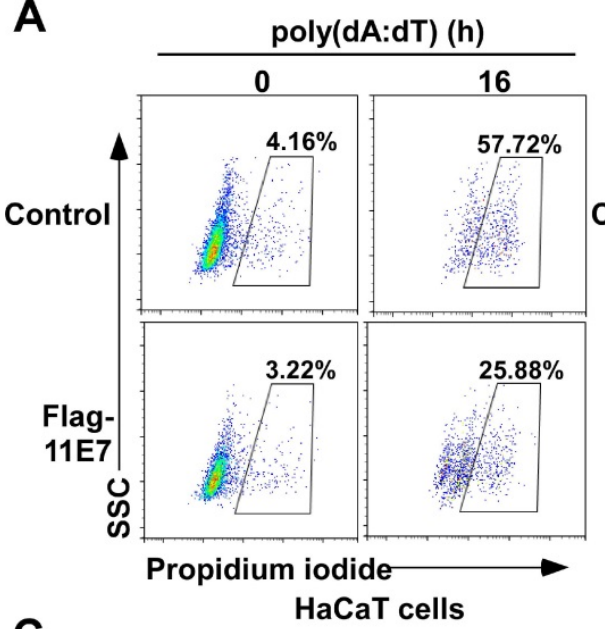

C

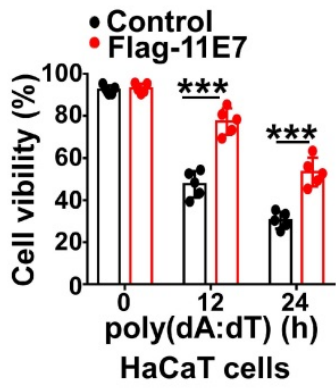

E

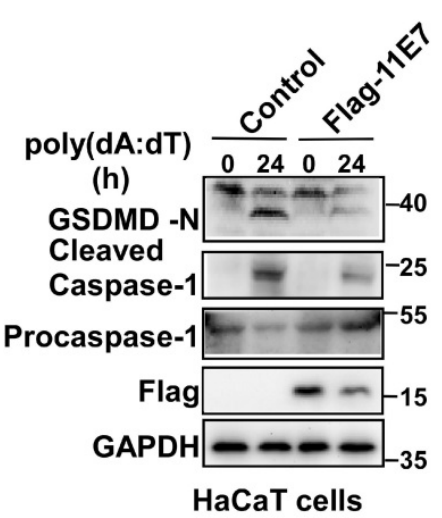

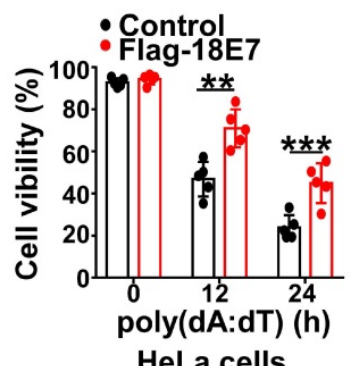

HeLa cells
poly(dA:dT) (h)

0

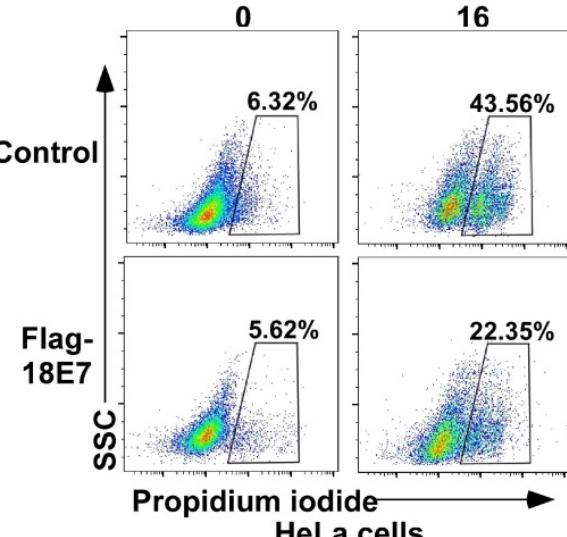

D

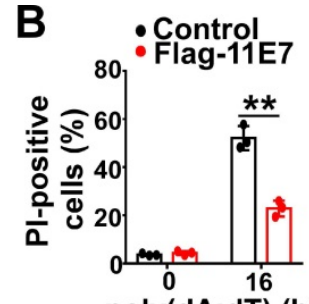

poly(dA:dT) (h)



poly(dA:dT) (h)
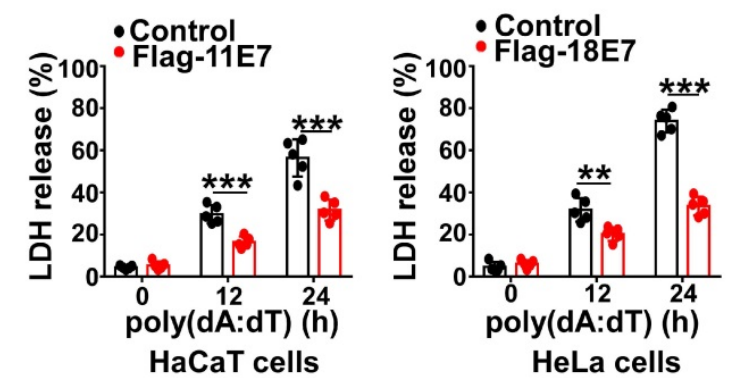

$\mathbf{F}$

Control

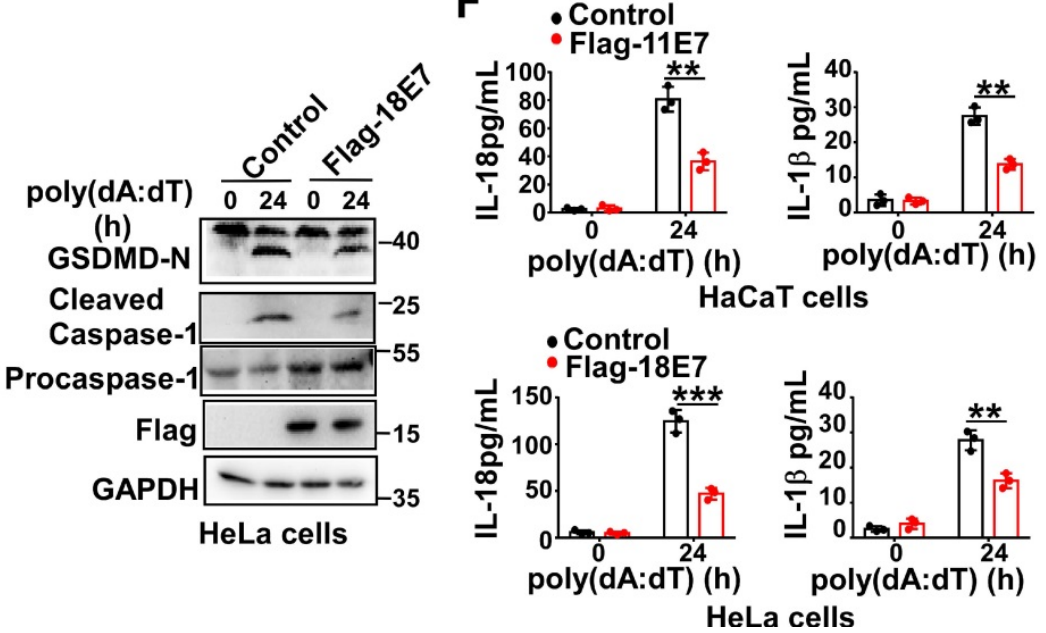

Figure 1. HPV E7 inhibits pyroptosis induced by intracellular dsDNA. (A-B) Flow cytometry analysis (A) and statistical analysis (B) of propidium iodide-positive HaCaT cells stably expressing HPV $11 E 7$ or HeLa cells stably expressing HPV 18 E7 after transfection with poly(dA:dT) for the indicated times. (C-D) Cell viability assay (C) and LDH assay (D) of $\mathrm{HaCaT}$ cells stably expressing HPV $11 \mathrm{E7}$ or HeLa cells stably expressing HPV $18 \mathrm{E} 7$ after transfection with poly(dA:dT) for the indicated times. (E) Immunoblot analysis of GSDMD and caspase-1 in the lysates of HaCaT cells stably expressing HPV 11 E7 or HeLa cells stably expressing HPV 18E7 after transfection with poly(dA:dT) for the indicated times. (F) ELISA analysis of IL-18 and IL-1 $\beta$ in HaCaT cells stably expressing HPV 11 E7 and HeLa cells stably expressing HPV 18 E7 after transfection with poly(dA:dT) for the indicated times. Data are presented as mean \pm SD of duplicate samples and are representative of at least three independent experiments. $P$ values are determined by two-tailed Student's $t$ test. $* * p<0.01$, *** $p<0.001$. 


\section{HPV E7 interacts with IFI16}

To explore the mechanisms by which HPV E7 inhibited cell pyroptosis, we first detected the mRNA levels of pyroptosis-associated molecules by RNAseq. There was no obvious difference in the mRNA levels of pyroptosis-associated genes between control $\mathrm{HaCaT}$ cells and HPV 11E7-overexpressing $\mathrm{HaCaT}$ cells (Figure S2A). Next, we investigated HPV 11E7-interacting proteins by immunoprecipitation (IP) and mass spectrometry. The information for the HPV 11E7-interacting proteins was shown in Supplementary Table 1. Among the HPV 11E7interacting proteins, we focused on the DNA sensor IFI16, which can assemble the IFI16 inflammasome and initiate cell pyroptosis (Figure 2A). To confirm the interaction between HPV 11E7 and IFI16, we immunoprecipitated the Flag epitope tag from the lysates of poly(dA:dT)-transfected cells over- expressing Flag-HPV 11E7, and immunoblot assay revealed that the interaction between HPV 11E7 and IFI16 was decreased by poly(dA:dT) transfection (Figure 2B). Additionally, the endogenous interaction between HPV 18E7 and IFI16 was reduced in HeLa cells after poly(dA:dT) transfection (Figure 2C). Co-IP and immunoblot assays showed that HPV 18E7 interacted with IFI16 in 293T cells which were transfected with plasmids for HPV 18E7 and IFI16 expression (Figure 2D). IFI16 contains PYD, HINA, HINB, and Neogenin C domains [31]. To identify which domain of IFI16 was responsible for the interaction with HPV E7, we constructed the mutant IFI16 plasmids (Figure 2E). Co-IP and western blot assays showed that the HINB domain of IFI16 interacted with HPV 18E7 (Figure 2F). These results strongly indicated that HPV E7 can interact with IFI16.



Figure 2. HPV E7 interacts with IFI16. (A) Mass spectrum data on IFI16 among HPV 11E7-interacting proteins identified by mass spectrometry. (B) Coimmunoprecipitation and immunoblot analysis of $\mathrm{HaCaT}$ cells stably expressing HPV $11 \mathrm{E7}$ after transfection with poly(dA:dT) for the indicated times. (C) Immunoblot analysis of HeLa cells transfected with poly(dA:dT) for the indicated times, followed by immunoprecipitation with IFI16, HPV 18E7 or immunoglobulin G (IgG)-conjugated magnetic beads. (D) Coimmunoprecipitation and immunoblot analysis of 293T cells cotransfected for $36 \mathrm{hr}$ with Flag-HPV 18 E7 and Myc-IFI16 or Flag-IFI16 and GFP-HPV 18 E7 plasmids followed by immunoprecipitation with anti-FlagM2 beads. (E) A schematic structure and IFI16 derivatives were shown. (F) Immunoblot analysis of $293 \mathrm{~T}$ cells cotransfected for 36 hr with GFP-HPV 18 E7 plus Flag-IFI16 or Flag-IFI16 mutant vectors, followed by immunoprecipitation with anti-FlagM2 beads. Data are representative of at least three independent experiments. 
A

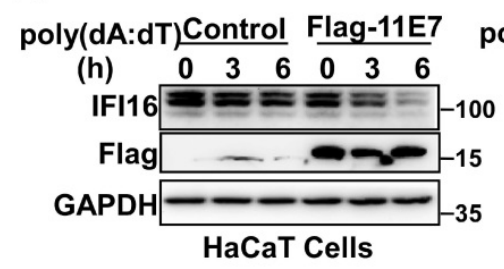

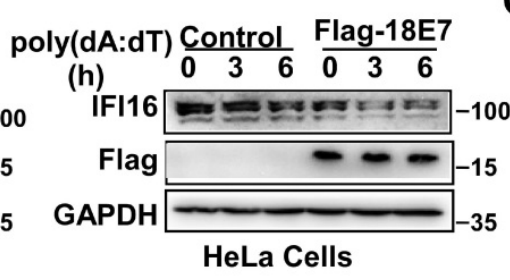

C



B

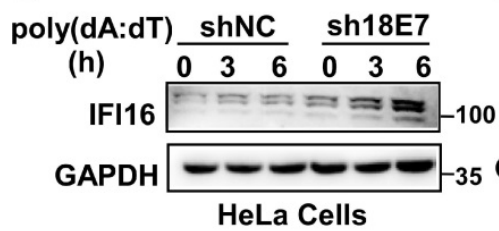

D

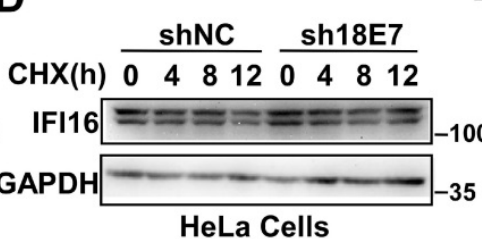



E

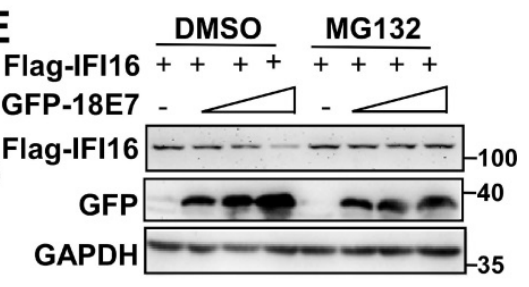

293T Cells

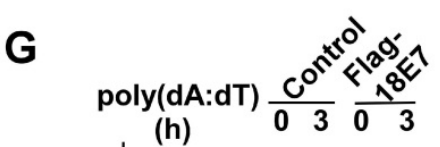

(h)

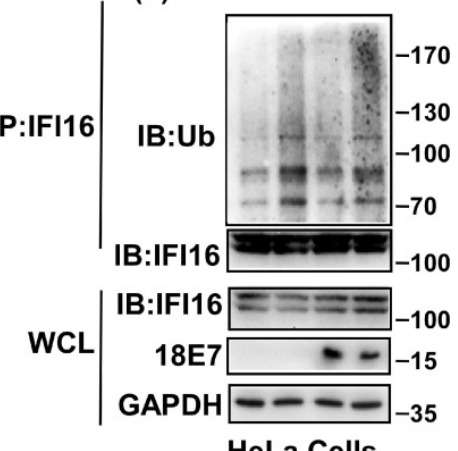

HeLa Cells

Figure 3. HPV E7 promotes the ubiquitin-proteasome-mediated degradation of IFII6. (A) Immunoblot analysis of HaCaT cells stably expressing HPV 11 E7 or HeLa cells stably expressing HPV 18 E7 transfected with poly(dA:dT) for the indicated times. (B) Immunoblot analysis of the lysates of HeLa cells transfected with shNC or sh18E7 plasmids for $36 \mathrm{hr}$ and transfected with poly(dA:dT) for the indicated times. (C) Immunoblot analysis of control HeLa cells or stably expressing HPV $18 \mathrm{E} 7 \mathrm{HeLa}$ cells treated with $\mathrm{CHX}(40 \mu \mathrm{g} / \mathrm{ml})$ for the indicated times after transfection with poly $(\mathrm{dA}: \mathrm{dT})$ for $1 \mathrm{hr}$. (D) Immunoblot analysis of IFI16 in the lysates of control HeLa cells or HPV $18 \mathrm{E} 7$-silenced HeLa cells treated with $\mathrm{CHX}(40 \mu \mathrm{g} / \mathrm{ml})$ for the indicated times after transfection with poly(dA:dT) for $1 \mathrm{hr}$. (E) Immunoblot analysis of $293 \mathrm{~T}$ cells cotransfected with the GFP-HPV 18 E7 and Flag-IFI16 vectors with or without MG132 treatment. (F) Immunoblot analysis of ubiquitinated IFI16 in control HeLa cells or HPV 18 E7-silenced HeLa cells were transfected with poly $(\mathrm{dA}: \mathrm{dT})$ for the indicated times and treated with MG132 for $6 \mathrm{hr}$ before cell harvest. Data are representative of at least three independent experiments. Data are representative of at least three independent experiments. (G) Immunoblot analysis of ubiquitinated IFI16 in control HeLa cells or HPV $18 \mathrm{E} 7$ knockout stable HeLa cells transfected with poly (dA:dT) for the indicated times and treated with MG132 for $6 \mathrm{hr}$ before cell harvest. Data are representative of at least three independent experiments.

\section{HPV E7 promotes the ubiquitin-proteasome- mediated degradation of IFII6}

The data described above showed that the interaction between HPV E7 and IFI16 was reduced in response to poly(dA:dT) transfection. Therefore, we hypothesized that the degradation of IFI16 by HPV E7 might cause the decreased interaction between HPV E7 and IFI16 after poly(dA:dT) transfection. To verify this hypothesis, we detected the expression of IFI16 by western blotting and found that overexpression of HPV 11E7 or HPV 18E7 promoted the degradation of IFI16 (Figure 3A) and knockdown of HPV 18E7 inhibited the degradation of IFI16 after poly(dA:dT) transfection (Figure 3B). The cycloheximide chase assay showed that overexpression of HPV $18 \mathrm{E} 7$ shortened the half-life of IFI16 (Figure 3C) and silencing of HPV 18E7 extended the half-life of the IFI16 protein in HeLa cells in response to poly(dA:dT) transfection (Figure 3D). To further confirm the role of HPV E7 in regulating the protein level of IFI16, we cotransfected 293T cells with plasmids for Flag-IFI16 and GFP-HPV 18E7 expression and observed that HPV 18E7 could also promote the degradation of IFI16 (Figure 3E). However, the degradation of IFI16 induced by HPV 18E7 was blocked by MG132, which indicated that the degradation of IFI16 induced by HPV E7 was dependent on the ubiquitin-proteasome system (Figure 3E). Next, we explored whether HPV 18E7 affected the ubiquitination of IFI16. HPV 18E7-silenced HeLa cells displayed decreased ubiquitination of IFI16 after poly(dA:dT) transfection (Figure 3F). We also found that overexpression of HPV 18E7 promoted the ubiquitination of IFI16 (Figure 3G). These data demonstrated that HPV E7 promotes the ubiquitin-mediated degradation of IFI16. 
A

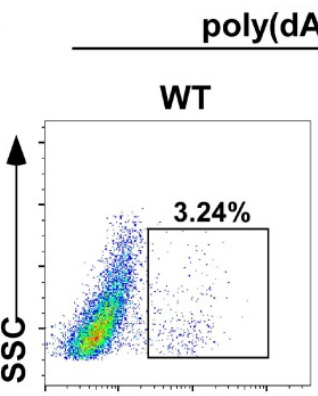

Propidium iodide
poly(dA:dT) 16h
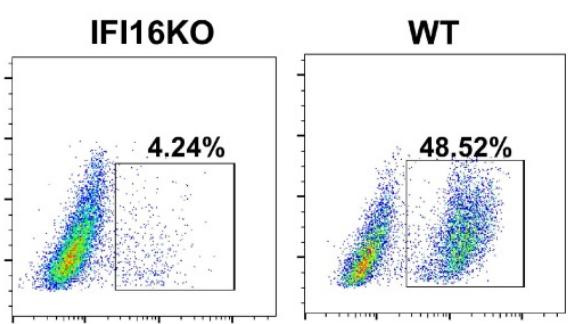

IFI16KO

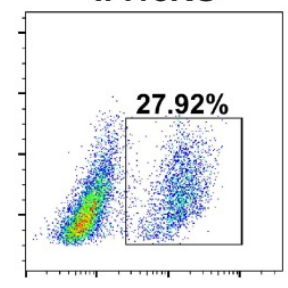

B



C $\cdot W T$
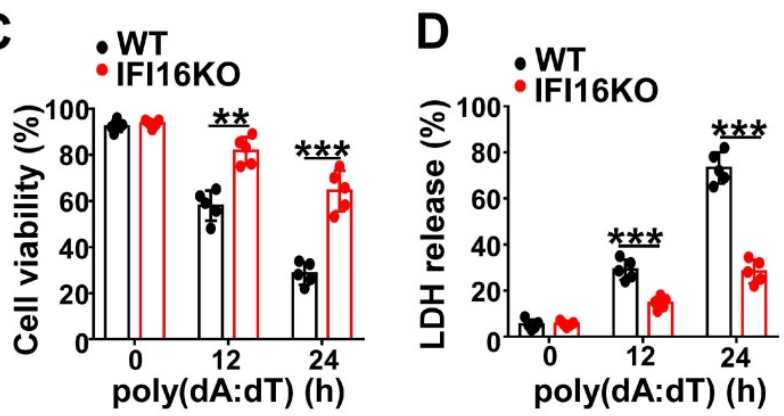

E

$\mathbf{F}$


Figure 4. IFI16 is responsible for pyroptosis induced by intracellular dsDNA. (A) Microscopy imaging showing cell death in IFI16 knockout stable HeLa cells or control HeLa cells transfected with poly(dA:dT) for $18 \mathrm{hr}$. (B) Flow cytometry analysis of propidium iodide-positive control HeLa cells or IFIl6 knockout stable HeLa cells transfected with poly $(\mathrm{dA}: \mathrm{dT})$ for $16 \mathrm{hr}$. Cell viability assay(C) and LDH assay (D) of control HeLa cells or IFI16 knockout stable HeLa cells transfected with poly(dA:dT) for the indicated times. (E) Immunoblot analysis of GSDMD and caspase-1 in the lysates of control HeLa cells or IFI16 knockout stable HeLa cells transfected with poly(dA:dT) for 24 hr. (F) ELISA analysis of IL-18 and IL-1 $\beta$ in control HeLa cells or IFI16 knockout stable HeLa cell transfected with poly(dA:dT) for 24 hr. Data are presented as mean \pm SD of duplicate samples and are representative of at least three independent experiments. $P$ values are determined by two-tailed Student's $t$ test. $* * p<0.01, * * * p<0.001$.

\section{IFI16 is responsible for the pyroptosis induced} by intracellular dsDNA

Next, we investigated whether IFI16 played a role in pyroptosis induced by intracellular dsDNA. Knockout of IFI16 by CRISPR/Cas9 significantly decreased the propidium iodide-positive HeLa cells after transfection with poly(dA:dT) (Figure 4A-B). IFI16KO HeLa cells displayed higher cell viability than WT HeLa cells (Figure 4C). However, the LDH level in IFI16KO HeLa cells was lower than that in WT HeLa cells after transfection with poly(dA:dT) (Figure 4D). Furthermore, knockout of IFI16 inhibited the cleavage of GSDMD and the activation of caspase-1 in HeLa cells (Figure 4E). ELISA assays showed that the levels of IL-1 $\beta$ and IL-18 were significantly decreased in IFI16KO HeLa cells transfected with poly(dA:dT) (Figure 4F). Taken together, our results demonstrated that IFI16 is responsible for the pyroptosis induced by intracellular dsDNA.

\section{HPV E7 interacts with IFII6 and E3 ligaseTRIM2 1}

Our results demonstrated that HPV E7 promoted the ubiquitin-proteasome-mediated degradation of IFI16. The ubiquitin-proteasome system is mainly composed of ubiquitin-activating enzyme (E1), ubiquitin-crosslinking enzyme (E2), ubiquitin ligase (E3) and the 26S proteasome [32]. Among these components, the E3 ubiquitin ligase is responsible for target protein recognition and mediates the degradation of substrates by the 26S proteasome [33]. 
To determine which E3 ubiquitin ligase mediated the degradation and ubiquitination of IFI16, we analyzed data from the above mass spectrometry experiment. Among the HPV 11E7-interacting proteins, the outstanding mascot scores indicated that TRIM21 could interact with HPV 11E7 (Figure 5A). To confirm the interaction between TRIM21, HPV E7, and IFI16, we immunoprecipitated Flag-HPV 11E7 from the lysates of cells overexpressing Flag-HPV 11E7; immunoblotting revealed that the interaction between HPV 11E7 and TRIM21 was increased in HaCaT cells after poly(dA:dT) transfection (Figure 5B). Additionally, the interaction between HPV 18E7 and TRIM21 was increased in HeLa cells during poly(dA:dT) transfection (Figure 5C). Further coimmunoprecipitation and immunoblot assays showed that HPV 18E7 and IFI16 interacted with TRIM21 in 293T cells transfected with plasmids expressing TRIM21, IFI16 or HPV 18E7 (Figure S3A-S3B). Additionally, HPV E7 promoted the interaction between IFI16 and TRIM21 (Figure S3C).

TRIM21 contains RING, BBOX, and PRY/SPRY domains [34]. To identify which domain of TRIM21 was responsible for the interaction with HPV E7 or IFI16, we constructed mutant TRIM21 plasmids and found that depletion of the PRY/SPRY domain eliminated the interactions of TRIM21 with HPV 18E7 and IFI16 (Figure 5D-5E). Furthermore, we found that TRIM21 interacted with the PYD domain of IFI16 (Figure 5F). These results strongly suggested that TRIM21 can interact with IFI16 and HPV E7.

\section{HPV E7 promotes the K33-linked ubiquitination and degradation of IFII 6 mediated by TRIM2I}

Next, we investigated whether TRIM21 mediated the ubiquitination and degradation of IFI16. The level of IFI16 was higher in TRIM21KO HeLa cells than in WT HeLa cells in response to poly(dA:dT) transfection (Figure 6A). Overexpression of TRIM21 promoted the degradation of IFI16 in HeLa cells after poly $(\mathrm{dA}: \mathrm{dT}$ ) transfection (Figure 6B). The cycloheximide chase assay showed that deletion of TRIM21 extended the half-life of the IFI16 protein (Figure 6C). Overexpression of TRIM21 shortened the half-life of IFI16 in HeLa cells (Figure S4A) or 293T cells (Figure S4B) transfected with poly(dA:dT). Furthermore, TRIM21 inhibited IFI16 protein levels when we cotransfected 293T cells with plasmids expressing Flag-IFI16 and Myc-TRIM21 (Figure 6D). Moreover, HPV 18E7 promoted the degradation of IFI16 mediated by TRIM21 (Figure 6E). However, this reduction in IFI16 mediated by TRIM21 was blocked by MG132, which implies that TRIM21 mediates the degradation of IFI16 by the ubiquitin-proteasome system (Figure 6F). Our data indicated that HPV E7 promotes the degradation of IFI16 mediated by TRIM21.

Next, we explored whether TRIM21 mediated the ubiquitination of IFI16. Knockout of TRIM21 deceased the ubiquitination of IFI16 in HeLa cells after poly(dA:dT) transfection (Figure 6G). The ubiquitination of IFI16 was increased in TRM21overexpressing HeLa cells after poly(dA:dT) transfection (Figure S4C). The TRIM21-mediated ubiquitination of IFI16 was also detected in 293T cells transfected with Flag-IFI16, HA-ub, and Myc-TRIM21 (Figure 6H). Furthermore, HPV 18E7 promoted the ubiquitination of IFI16 mediated by TRIM21 (Figure 6I). Ubiquitin contains seven lysine (K) residues (K6, $\mathrm{K} 11, \mathrm{~K} 27, \mathrm{~K} 29, \mathrm{~K} 33, \mathrm{~K} 48$, and $\mathrm{K} 63$ ) that are utilized to form ubiquitination chains [35]. To determine which form of polyubiquitin chain was attached to IFI16 by TRIM21, we mutated each of the Lys residues on ubiquitin and tested their effects on IFI16 ubiquitination in 293T cells overexpressing TRIM21 and IFI16. Only K33R ubiquitin did not support TRIM21-mediated IFI16 ubiquitination (Figure 6J). We next used another set of ubiquitin mutants in which all but one Lys residue were replaced with an Arg. Among these ubiquitin mutants, only K33-ubiquitin and wild-type ubiquitin promoted ubiquitin chain formation on IFI16 (Figure 6K). Taken together, our results demonstrated that HPV E7 can promote the K33-linked ubiquitination and degradation of IFI16 mediated by TRIM21.

\section{TRIM21 downregulates cell pyroptosis induced by poly(dA:dT)}

Next, we investigated whether TRIM21 could regulate pyroptosis induced by poly(dA:dT). Flow cytometry also showed that more TRIM21 KO HeLa cells than control cells were propidium iodidepositive in response to transfection with poly(dA:dT) (Figure 7A-B). In addition, TRIM21KO HeLa cells displayed decreased cell viability compared to WT HeLa cells in response to poly(dA:dT) (Figure 7C). Furthermore, knockout of TRIM21 significantly promoted the release of LDH in HeLa cells (Figure 7D). Moreover, immunoblotting showed that the level of GSDMD-N and activated caspase-1 were higher in TRIM21KO HeLa cells than in WT HeLa cells after transfection with poly(dA:dT) (Figure 7E). Knockout of TRIM21 significantly enhanced the production of IL-1 $\beta$ and IL-18 in HeLa cells transfected with poly(dA:dT) (Figure 7F). Thus, our results indicated that TRIM21 inhibits pyroptosis induced by $\operatorname{poly}(\mathrm{dA}: \mathrm{dT})$. 
A

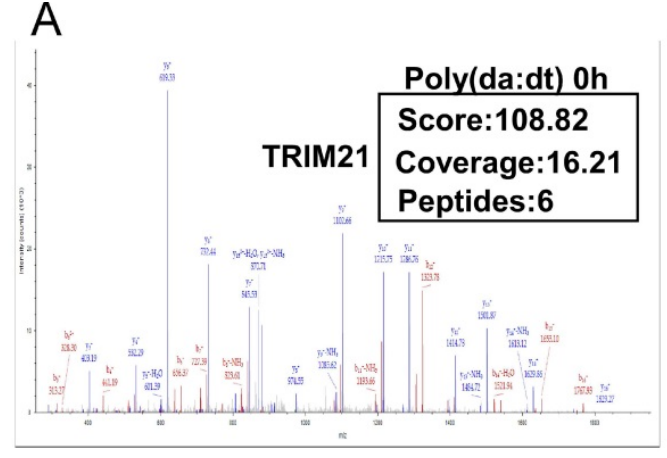

C

IP:IFI16

IP:TRIM21

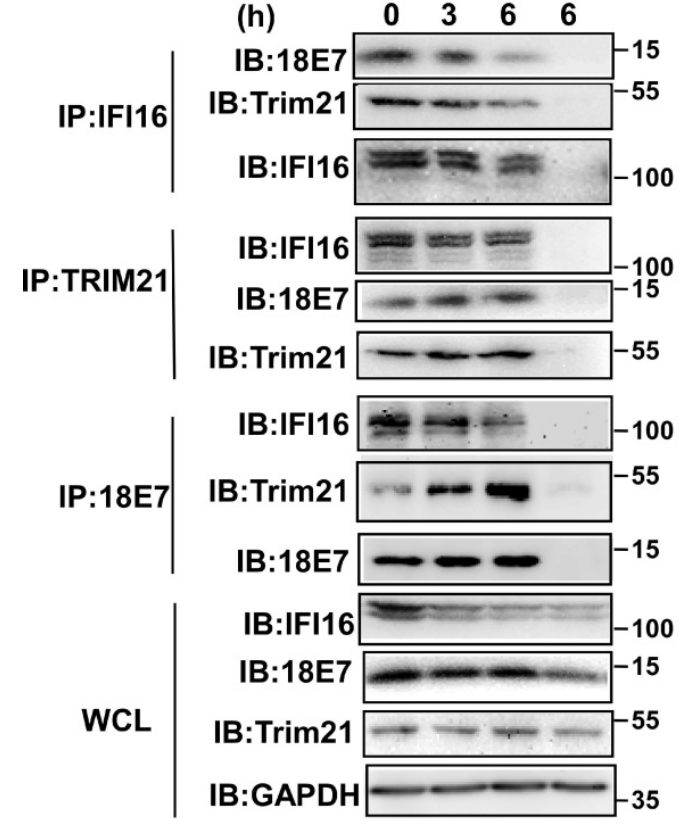

B

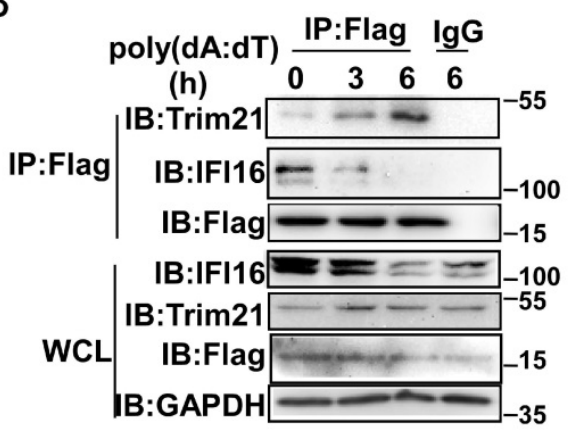

D

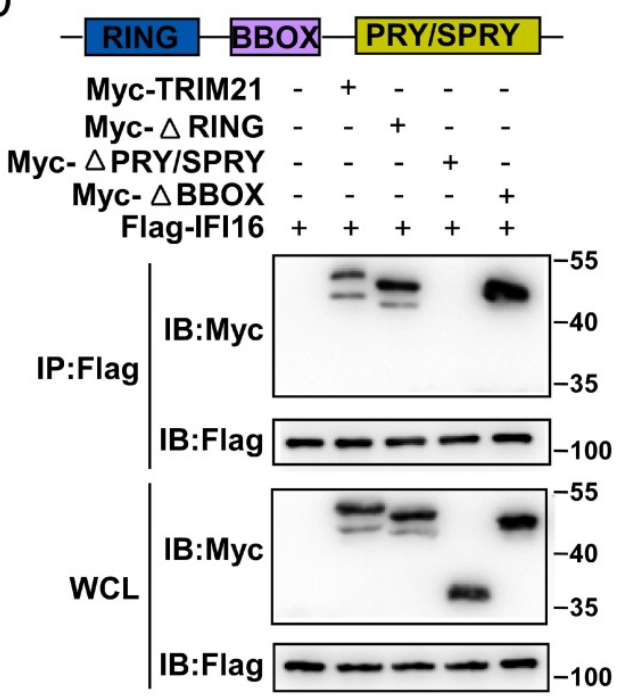

$E$

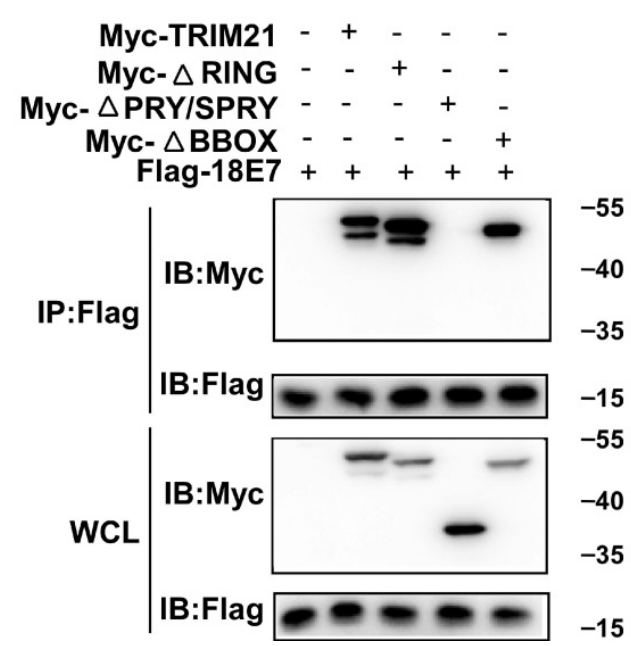

F

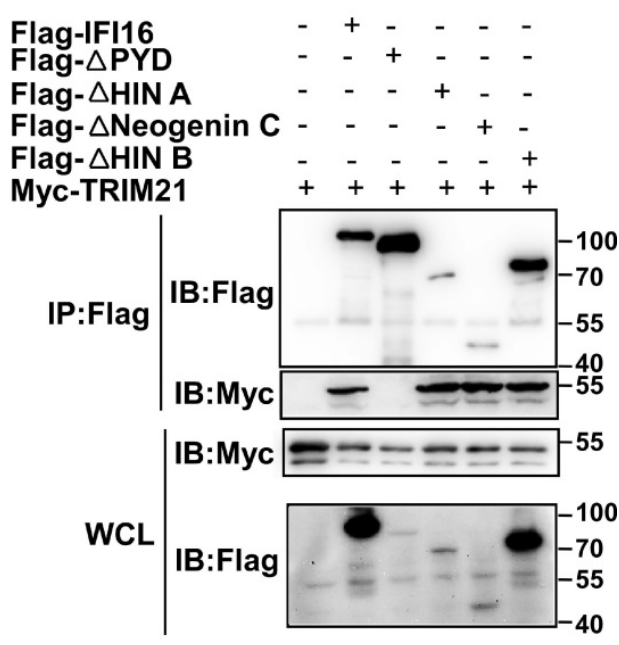

Figure 5. HPV E7 interacts with IFI16 and the E3 ligaseTRIM21. (A) Mass spectrum data of TRIM21 among HPV 11 E7-interacting proteins identified by mass spectrometry. (B) Immunoblot analysis of HaCaT cells stably expressing HPV 11 E7 transfected with poly(dA:dT) for the indicated times, followed by immunoprecipitation with anti-FlagM2 beads. (C) Immunoblot analysis of HeLa cells transfected with poly(dA:dT) for the indicated times, followed by immunoprecipitation with IFI16, HPV 18E7, TRIM21 or immunoglobulin G (IgG)-conjugated magnetic beads. (D) Coimmunoprecipitation and immunoblot analysis of $293 \mathrm{~T}$ cells cotransfected for 36 hr with Flag-IFI16 plus Myc-TRIM21 or Myc-TRIM21 mutant vectors, followed by immunoprecipitation with anti-Flag-M2 beads. (E) Coimmunoprecipitation and immunoblot analysis of 293 T cells cotransfected for $36 \mathrm{hr}$ with Flag-HPV $18 \mathrm{E} 7$ plus Myc-TRIM21 or Myc-TRIM21 mutant vectors, followed by immunoprecipitation with anti-FlagM2 beads. (F) Coimmunoprecipitation and immunoblot analysis of $293 \mathrm{~T}$ cells cotransfected for $36 \mathrm{hr}$ with Myc-TRIM21 and Flag-IFI16 or Flag-IFII6 mutant vectors, followed by immunoprecipitation with anti-FlagM2 beads. 
A

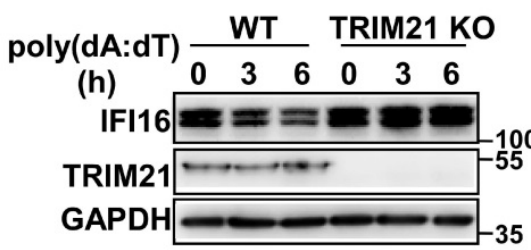

HeLa Cells
C

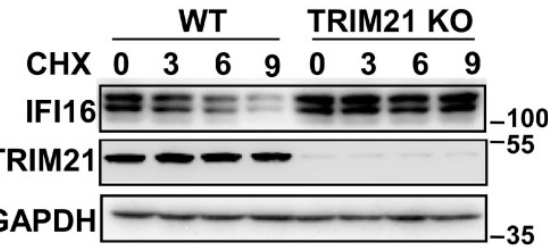

HeLa Cells
D

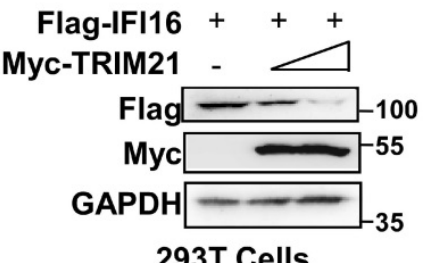

293T Cells
B

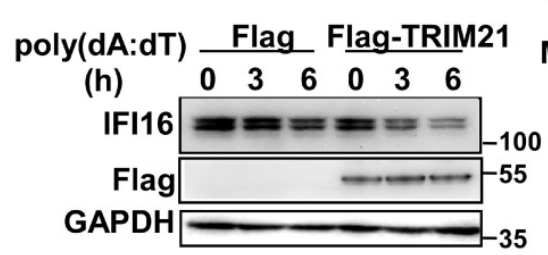

HeLa Cells
E

Flag-IFI16 ++++

$\mathbf{F}$

Myc-TRIM21 - + + +

GFP-18E7 - -

Flag $-\div-\cdots$

Myc -55

GFP -40

GAPDH $-\ldots-{ }_{35}$

293T Cells

F

G

$\operatorname{poly}(d A: d T) \quad \begin{array}{llll}0 & 3 & & 3\end{array}$

(h)

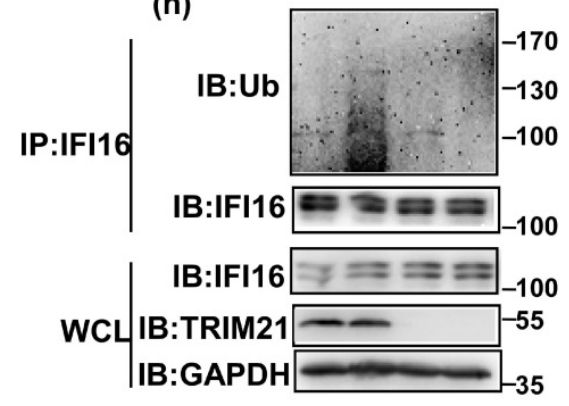

H

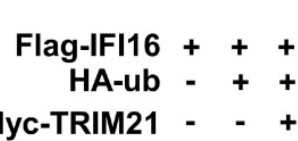

IP:Flag

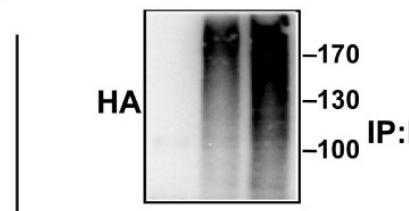

Flag $=-\dot{m}-100$

WCL $\mid \begin{aligned} & \text { Myc } \\ & \text { IB:Flag } \\ & \text { GAPDH }\end{aligned}$
MG132

DMSO
Flag-IFI16 ++++++++

Myc-TRIM21 - $\square$ -

Flag-IFI16 - - - - 100

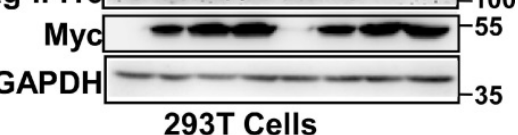

J

K

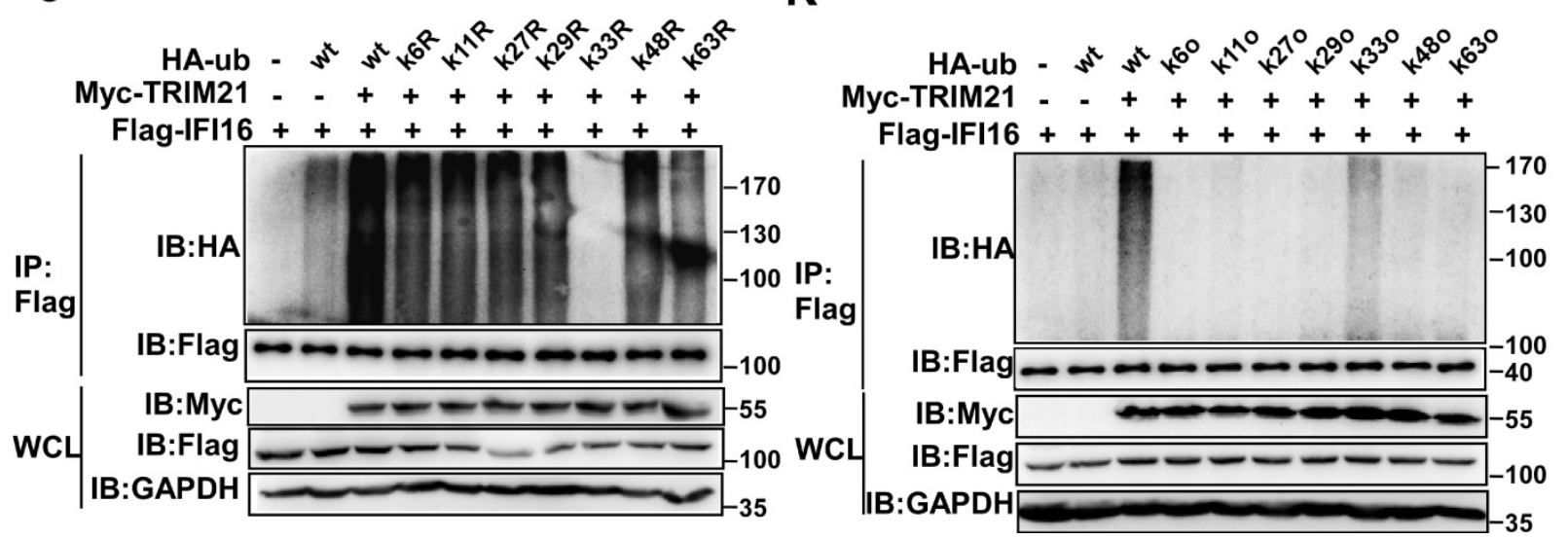

Figure 6. HPV E7 promotes the K33-linked ubiquitination and degradation of IFI16 mediated by TRIM21. (A) Immunoblot analysis of lysates in control HeLa cells or TRIM21 knockout stable HeLa cells transfected with poly(dA:dT) for the indicated times. (B) Immunoblot analysis of IFI16 in the lysates of TRIM21 knockout stable HeLa cells treated with $\mathrm{CHX}(40 \mu \mathrm{g} / \mathrm{ml})$ for the indicated number of hours after transfection with poly(dA:dT) for 1 hr. (C) Immunoblot analysis of $293 \mathrm{~T}$ cells cotransfected with the Myc-TRIM21 and Flag-IFI16 vectors for 36 hr. (D) Immunoblot analysis of 293T cells cotransfected with the Myc-TRIM21 and Flag-IFI16 vectors with or without MG132 treatment. (E) Immunoblot analysis of 293T cells cotransfected with the Myc-TRIM21 and Flag-IFI16 vectors treated with $\mathrm{CHX}(40 \mu \mathrm{g} / \mathrm{ml})$ for the indicated number of hours. (F) Immunoblot analysis of 293T cells cotransfected with Myc-TRIM2l and the Flag-IFI1 6 or GFP-HPV 18 E7 vector for $36 \mathrm{hr}$. (G) Immunoblot analysis of ubiquitinated IFI16 in control HeLa cells or TRIM21 knockout stable HeLa cells transfected with poly(dA:dT) for the indicated times and treated with MG132 for 6 hr before cell harvest. (H) Immunoblot analysis of ubiquitinated IFI16 in 293T cells cotransfected with Myc-TRIM21 and the Flag-IFI16 or HA-ub vector for $36 \mathrm{hr}$ with or without MG132 treatment for 6 hr before cell harvest. (I) Immunoblot analysis of ubiquitinated IFI16 in 293T cells transfected with combinations of the Myc-TRIM21, Flag-IFI16, HA-ub, and GFP-HPV 18 E7 vectors for 36 hr and treated with MG132 for 6 hr before cell harvest. (J) Immunoblot analysis of ubiquitinated IFI16 in 293T cells transfected with combinations of the Myc-TRIM21, Flag-IFI16, HA-ub, and HA-ub mutant (each of the Lys residues were replaced by an Arg residue) vectors for $36 \mathrm{hr}$ and treated with MG132 for 6 hr before cell harvest. (K) Immunoblot analysis of ubiquitinated IFI16 in 293T cells transfected with combinations of the Myc-TRIM21, Flag-IFI16, HA-ub, HA-ub mutant (all Lys residues but one was replaced with Arg residues) vectors for $36 \mathrm{hr}$ and treated with MG132 for $6 \mathrm{hr}$ before cell harvest. Data are representative of at least three independent experiments. 



E

$\mathbf{F}$
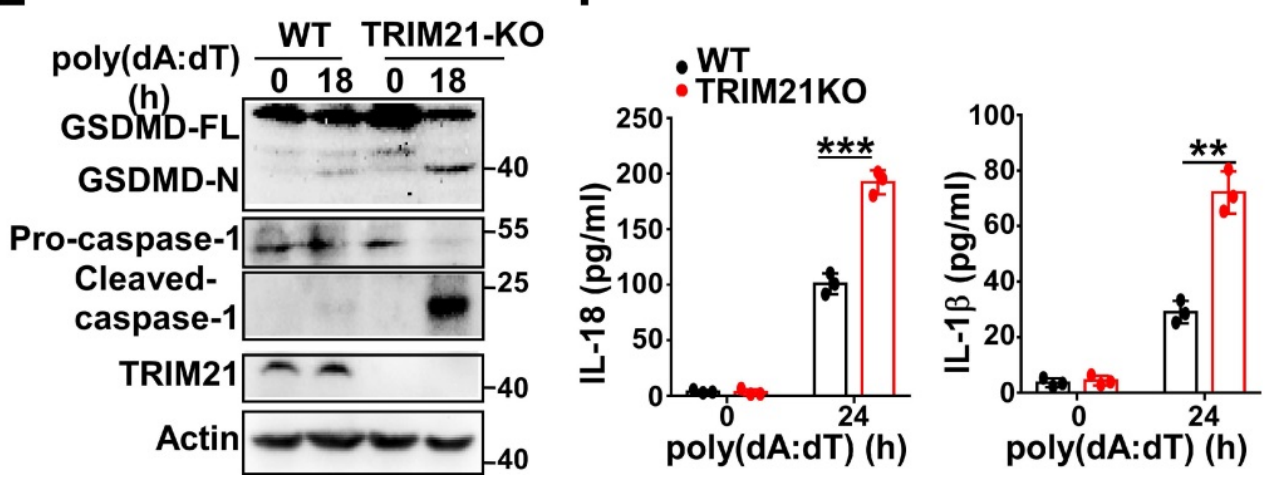

Figure 7. TRIM21 downregulates cell pyroptosis induced by poly(dA:dT). (A) Microscopy imaging of cell death in control HeLa cells or TRIM21 knockout stable HeLa cells transfected with poly(dA:dT) for $18 \mathrm{hr}$. (B) Flow cytometry analysis of propidium iodide-positive control HeLa cells or TRIM21 knockout stable HeLa cells transfected with poly (dA:dT) for $16 \mathrm{hr}$. (C) Cell viability assay in control HeLa cells or TRIM21 knockout stable HeLa cells transfected with poly(dA:dT) for the indicated times. (D) LDH assay in control HeLa cells or TRIM21 knockout stable HeLa cells transfected with poly(dA:dT) for the indicated times. (E) Immunoblot analysis of GSDMD and caspase-1 in the lysates of control HeLa cells or TRIM21 knockout stable HeLa cells transfected with poly(dA:dT) for $24 \mathrm{hr}$. (F) ELISA analysis of IL-18 and IL-1 $\beta$ in control HeLa cells or knockout stable HeLa cells transfected with poly $(\mathrm{dA}: \mathrm{dT})$ for $24 \mathrm{hr}$. Data are presented as mean \pm SD of duplicate samples and are representative of at least three independent experiments. $\mathrm{P}$ values are determined by two-tailed Student's $t$ test. $* * p<0.01$, $* * * p<0.001$.

\section{Discussion}

Host cell death, including apoptotic cell death, necrotic cell death, and pyroptotic cell death, is a critical immune defense mechanism in response to infection with pathogenic organisms [36]. However, HPV has evolved mechanisms to manipulate host cell death to enhance its ability to survive, evade immunosurveillance and cause disease [37, 38]. For example, the HPV E5 protein can protect cells from tumor necrosis factor-related apoptosis [39], HPV E6 can inhibit apoptosis mainly through the p53 pathway
[40], and HPV E7 plays a role in both apoptosis activation and inhibition [41]. However, whether HPV can regulate cell pyroptosis is unknown. Here, we found that HPV 11E7 or HPV 18E7 could inhibit the activation of caspase-1 and cleavage of GSDM-D. HPV E7 could also attenuate cell swelling and the production of IL-18 and IL- $1 \beta$ which can promote the production of proinflammatory cytokines from immune cells and enhance the expansion, migration, and activation of macrophages, neutrophils, $\mathrm{CD} 8^{+} \mathrm{T}$ cells, Th17 cells and NK cells during infections [42]. Therefore, HPV could evade immunosurveillance and 
cause cervical cancer or condyloma acuminate by regulating cell pyroptosis. Our data reveals one of the mechanisms of HPV immune escape.

IFI16 is required for the innate immune response to transfected DNA and infection with nuclear and cytosolic DNA viruses [24, 43]. IFI16 and cGAS cooperate to sense intracellular DNA and enable the optimal production of cGAMP, which activates the STING-TBK1 pathway and induces the production of type I interferon in human keratinocytes or macrophages [23, 24]. IFI16 can detect viral DNA, initiate assembly of the inflammasome and mediate cell pyroptosis upon infection with DNA viruses, such as KSHV, EBV or HSV-1 [44]. IFI16 inhibits HPV18 replication by repressing viral gene expression and replication [45]. Numerous studies have shown that pathogens promote immune escape by regulating the expression and function of IFI16 through mechanisms such as production of the HSV-1-encoded infected cell protein (ICP)0 protein, which possesses E3 ubiquitin ligase activity and mediates the degradation of IFI16 by the proteasome [28]. KSHV selectively degrades IFI16 to evade the host immune system to maintain latency [46]. The HCMV major tegument protein pUL83 interacts with IFI16 and inhibits the oligomerization of activated IFI16, resulting in diminished signal transmission from IFI16 to the STING-TBK1-IRF3 signaling pathway [47]. Our results demonstrated that HPV E7 interacted with the HINB domain of IFI16 and promoted the ubiquitin-proteasome-mediated degradation of IFI16 by recruiting the E3 ligase TRIM21, resulting in the inhibition of cell pyroptosis during infection.

Ubiquitination is a dynamic, multifaceted posttranslational modification that plays a pivotal role in the regulation of many cellular processes including proteasomal degradation, the cell cycle, DNA metabolism, and various signal transduction pathways [48]. Many viruses including HPV usurp ubiquitination and ubiquitin-like modifications to reprogram the cellular environment to favor viral persistence and reproduction [49]. High-risk HPV E6 protein is the prototype of HPV-mediated modulation of the host cell through recruiting the ubiquitin ligase E6AP to mediate the ubiquitination and degradation of p53 [50]. HPV16 E7 promotes the ubiquitination and degradation of $\mathrm{pRB}$ mediated by the ubiquitin ligase cullin 2 and the E6-pRb complex [51]. High-risk HPV E7 limits keratinocyte differentiation and contributes to HPV-mediated oncogenesis by facilitating E3 ligase UBR4-mediated ubiquitination and the degradation of PTPN14 [52]. The HPV E6 and E7 suppress IRF3 activation by inhibiting K63-linked ubiquitination of TRAF3 through upregulating
UCHL1 and inhibiting the immune response upon HPV infection [53]. Here, we found that the interaction between HPV E7 and TRIM21 was increased after transfection with poly(dA:dT). It has been reported that the phosphorylation of TRIM21 can be catalyzed by IKK $\beta$ or TBK1 kinases, thus releasing the auto-inhibitory mechanism of TRIM21 [54]. Therefore, the increased interaction of HPV E7 and TRIM21 may be due to the increased phosphorylation of TRIM21 catalyzed by IKK $\beta$ or TBK1 kinases upon poly(dA:dT) transfection. We also found that the interaction between HPV E7, TRIM21 and IFI16 was significantly decreased after transfection with poly(dA:dT). This implied that HPV E7 could recruit the E3 ligase TRIM21 to mediate the ubiquitination and degradation of IFI16 during HPV infection.

The E3 ligase TRIM21 is a member of the TRIM protein family largely involved in innate immunity [55]. TRIM21 mediates the polyubiquitination and degradation of the DNA sensor DDX41 and the IRF transcription factors IRF3, IRF5, and IRF7 to negatively regulate the production of interferon- $\beta$ during viral infection [56]. Recent research reported that TRIM21 can also promote the STING-mediated IFI16 degradation and negatively regulate the production of type I interferon [57]. However, a growing number of studies have shown that TRIM21 positively upregulates type I IFN signaling through promoting cGAS and RIG-I sensing of viral genomes or interfering with the degradation of IRF3 and IRF8 $[58,59]$. Therefore, the role of TRIM21 in antiviral innate immunity remains controversial, and it is unclear whether TRIM21 plays a role in inflammasome-mediated cell pyroptosis. In this study, we found the proportion of swollen cells, the level of GSDMD-N and the activated caspase-1 were higher in TRIM21KO HeLa cells than in WT HeLa cells after transfection with poly(dA:dT). Knockout of TRIM21 significantly inhibited the production of IL-1 $\beta$ and IL-18. Moreover, the regulatory effect of HPV 18E7 on pyroptosis and the stability of IFI16 were absent in TRIM21 KO HeLa cells transfected with poly $(\mathrm{dA}: \mathrm{dT})$. Our results indicated that TRIM21 negatively regulated the activation of the IFI16 inflammasome and that HPV E7-mediated regulation of pyroptosis was dependent on TRIM21. Therefore, based on the results of our study, we have schematically drawn a diagraph to show the effect and mechanisms of HPV E7 on cell pyroptosis (Figure 8).

\section{Supplementary Material}

Supplementary figures and tables.

http://www.ijbs.com/v16p2924s1.pdf 


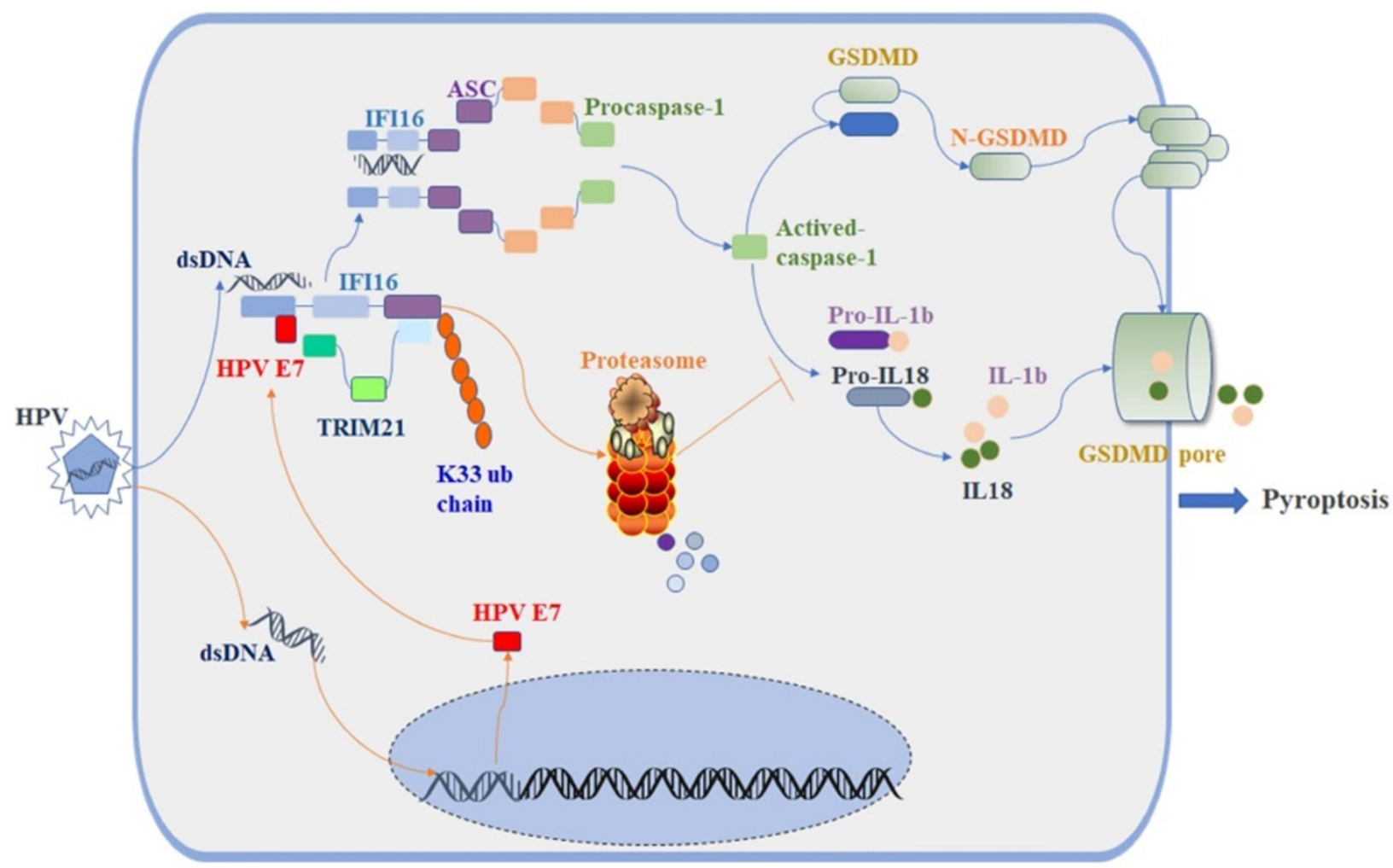

Figure 8. Schematic diagram showing that HPV E7 interacted with IFI16 and promoted the ubiquitin-mediated degradation of IFI16 by recruiting the E3 ligase TRIM21, resulting in the inhibition of cell pyroptosis during HPV infection.

\section{Acknowledgements}

This work was supported by grants from the National Natural Science Foundation of China (81071302, 81472889, 81573057, 81703135, and 81801992), the National Health and Family Planning Commission Foundation of China (2015117502), the Natural Science Foundation of Zhejiang Province (LY19H190006 and LQ19H190002) and the Medical and Health Science and Technology Project of Zhejiang province (2018256428). All authors have read and approved the final manuscript.

\section{Competing Interests}

The authors have declared that no competing interest exists.

\section{References}

1. Clarke MA, Cheung LC, Lorey T, Hare B, Landy R, Tokugawa D, et al. Five-year prospective evaluation of cytology, HPV testing, and biomarkers for detection of anal precancer in HIV+ MSM. Clin Infect Dis. 2018.

2. Patel P, Bush T, Conley L, Unger ER, Darragh TM, Henry K, et al. Prevalence, Incidence, and Clearance of Human Papillomavirus Types Covered by Current Vaccines in Men With Human Immunodeficiency Virus in the SUN Study. J Infect Dis. 2019.

3. Sasagawa $\mathrm{T}, \mathrm{Takagi} \mathrm{H}$, Makinoda $\mathrm{S}$. Immune responses against human papillomavirus (HPV) infection and evasion of host defense in cervical cancer. J Infect Chemother. 2012; 18: 807-15.

4. Taberna M, Mena M, Pavon MA, Alemany L, Gillison ML, Mesia R. Human papillomavirus-related oropharyngeal cancer. Ann Oncol. 2017; 28: 2386-98.

5. Stanley MA, Pett MR, Coleman N. HPV: from infection to cancer. Biochem Soc Trans. 2007; 35: 1456-60.

6. Munguia-Moreno JA, Diaz-Chavez J, Garcia-Villa E, Albino-Sanchez ME, Mendoza-Villanueva D, Ocadiz-Delgado R, et al. Early synergistic interactions between the HPV16E7 oncoprotein and 17beta-oestradiol for repressing the expression of Granzyme B in a cervical cancer model. Int J Oncol. 2018; 53: 579-91.

7. Park JS, Kim EJ, Kwon HJ, Hwang ES, Namkoong SE, Um SJ. Inactivation of interferon regulatory factor-1 tumor suppressor protein by HPV E7 oncoprotein. Implication for the E7-mediated immune evasion mechanism in cervical carcinogenesis. J Biol Chem. 2000; 275: 6764-9.

8. Richards $\mathrm{KH}$, Doble R, Wasson CW, Haider M, Blair GE, Wittmann M, et al. Human papillomavirus E7 oncoprotein increases production of the antiinflammatory interleukin-18 binding protein in keratinocytes. J Virol. 2014; 88: 4173-9.

9. Gautam D, Johnson BA, Mac M, Moody CA. SETD2-dependent H3K36me3 plays a critical role in epigenetic regulation of the HPV31 life cycle. PLoS Pathog. 2018; 14: e1007367.

10. Cicchini L, Blumhagen RZ, Westrich JA, Myers ME, Warren CJ, Siska C, et al. High-Risk Human Papillomavirus E7 Alters Host DNA Methylome and Represses HLA-E Expression in Human Keratinocytes. Sci Rep. 2017; 7: 3633.

11. de la Cruz-Hernandez E, Perez-Cardenas E, Contreras-Paredes A, Cantu D, Mohar A, Lizano M, et al. The effects of DNA methylation and histone deacetylase inhibitors on human papillomavirus early gene expression in cervical cancer, an in vitro and clinical study. Virol J. 2007; 4: 18.

12. Jakubickova L, Barathova M, Pastorekova S, Pastorek J, Gibadulinova A. Expression of S100P gene in cervical carcinoma cells is independent of E7 human papillomavirus oncogene. Acta Virol. 2005; 49: 133-7.

13. Lau L, Gray EE, Brunette RL, Stetson DB. DNA tumor virus oncogenes antagonize the cGAS-STING DNA-sensing pathway. Science. 2015; 350: 568-71.

14. Hong S, Mehta KP, Laimins LA. Suppression of STAT-1 expression by human papillomaviruses is necessary for differentiation-dependent genome amplification and plasmid maintenance. J Virol. 2011; 85: 9486-94.

15. Lee SH, Kim JW, Lee HW, Cho YS, Oh SH, Kim YJ, et al. Interferon regulatory factor-1 (IRF-1) is a mediator for interferon-gamma induced attenuation of telomerase activity and human telomerase reverse transcriptase (hTERT) expression. Oncogene. 2003; 22: 381-91.

16. Yuan J, Najafov A, Py BF. Roles of Caspases in Necrotic Cell Death. Cell. 2016; 167: 1693-704.

17. Daniels BP, Snyder AG, Olsen TM, Orozco S, Oguin TH, 3rd, Tait SWG, et al RIPK3 Restricts Viral Pathogenesis via Cell Death-Independent Neuroinflammation. Cell. 2017; 169: 301-13 e11.

18. Shi J, Zhao Y, Wang K, Shi X, Wang Y, Huang H, et al. Cleavage of GSDMD by inflammatory caspases determines pyroptotic cell death. Nature. 2015; 526: $660-5$.

19. Ding J, Wang K, Liu W, She Y, Sun Q, Shi J, et al. Pore-forming activity and structural autoinhibition of the gasdermin family. Nature. 2016; 535: 111-6. 
20. Liu X, Zhang Z, Ruan J, Pan Y, Magupalli VG, Wu H, et al. Inflammasomeactivated gasdermin $D$ causes pyroptosis by forming membrane pores. Nature. 2016; 535: 153-8.

21. Broz P. Immunology: Caspase target drives pyroptosis. Nature. 2015; 526: 642-3.

22. Strowig T, Henao-Mejia J, Elinav E, Flavell R. Inflammasomes in health and disease. Nature. 2012; 481: 278-86.

23. Almine JF, O'Hare CA, Dunphy G, Haga IR, Naik RJ, Atrih A, et al. IFI16 and cGAS cooperate in the activation of STING during DNA sensing in human keratinocytes. Nat Commun. 2017; 8: 14392.

24. Jonsson KL, Laustsen A, Krapp C, Skipper KA, Thavachelvam K, Hotter D, et al. IFI16 is required for DNA sensing in human macrophages by promoting production and function of cGAMP. Nat Commun. 2017; 8: 14391.

25. Roy A, Dutta D, Iqbal J, Pisano G, Gjyshi O, Ansari MA, et al. Nuclear Innate Immune DNA Sensor IFI16 Is Degraded during Lytic Reactivation of Kaposi's Sarcoma-Associated Herpesvirus (KSHV): Role of IFI16 in Maintenance of KSHV Latency. J Virol. 2016; 90: 8822-41.

26. Dutta D, Dutta S, Veettil MV, Roy A, Ansari MA, Iqbal J, et al. BRCA1 Regulates IFI16 Mediated Nuclear Innate Sensing of Herpes Viral DNA and Subsequent Induction of the Innate Inflammasome and Interferon-beta Responses. PLoS Pathog. 2015; 11: e1005030.

27. Torii Y, Kawada JI, Murata T, Yoshiyama H, Kimura H, Ito Y. Epstein-Barr virus infection-induced inflammasome activation in human monocytes. PLoS One. 2017; 12: e0175053.

28. Orzalli MH, DeLuca NA, Knipe DM. Nuclear IFI16 induction of IRF-3 signaling during herpesviral infection and degradation of IFI16 by the viral ICP0 protein. Proc Natl Acad Sci U S A. 2012; 109: E3008-17.

29. Galluzzi L, Vitale I, Aaronson SA, Abrams JM, Adam D, Agostinis P, et al. Molecular mechanisms of cell death: recommendations of the Nomenclature Committee on Cell Death 2018. Cell Death Differ. 2018; 25: 486-541.

30. Monroe KM, Yang Z, Johnson JR, Geng X, Doitsh G, Krogan NJ, et al. IFI16 DNA sensor is required for death of lymphoid CD4 T cells abortively infected with HIV. Science. 2014; 343: 428-32.

31. Liao JC, Lam R, Brazda V, Duan S, Ravichandran M, Ma J, et al. Interferon-inducible protein 16: insight into the interaction with tumor suppressor p53. Structure. 2011; 19: 418-29.

32. Li X, Elmira E, Rohondia S, Wang J, Liu J, Dou QP. A patent review of the ubiquitin ligase system: 2015-2018. Expert Opin Ther Pat. 2018; 28: 919-37.

33. Wertz IE, Wang X. From Discovery to Bedside: Targeting the Ubiquitin System. Cell Chem Biol. 2019; 26: 156-77.

34. James LC, Keeble AH, Khan Z, Rhodes DA, Trowsdale J. Structural basis for PRYSPRY-mediated tripartite motif (TRIM) protein function. Proc Natl Acad Sci U S A. 2007; 104: 6200-5.

35. Park S, Krist DT, Statsyuk AV. Protein ubiquitination and formation of polyubiquitin chains without ATP, E1 and E2 enzymes. Chem Sci. 2015; 6: 1770-9.

36. Cambier CJ, Falkow S, Ramakrishnan L. Host evasion and exploitation schemes of Mycobacterium tuberculosis. Cell. 2014; 159: 1497-509.

37. Baruah P, Bullenkamp J, Wilson POG, Lee M, Kaski JC, Dumitriu IE. TLR9 Mediated Tumor-Stroma Interactions in Human Papilloma Virus (HPV)Positive Head and Neck Squamous Cell Carcinoma Up-Regulate PD-L1 and PD-L2. Front Immunol. 2019; 10: 1644

38. Bogani G, Leone Roberti Maggiore U, Signorelli M, Martinelli F, Ditto A, Sabatucci I, et al. The role of human papillomavirus vaccines in cervical cancer: Prevention and treatment. Crit Rev Oncol Hematol. 2018; 122: 92-7.

39. Liao S, Deng D, Zhang W, Hu X, Wang W, Wang H, et al. Human papillomavirus 16/18 E5 promotes cervical cancer cell proliferation, migration and invasion in vitro and accelerates tumor growth in vivo. Oncol Rep. 2013; 29: 95-102.

40. Seltzsam S, Ziemann F, Dreffke K, Preising S, Arenz A, Schotz U, et al. In HPV-Positive HNSCC Cells, Functional Restoration of the p53/p21 Pathway by Proteasome Inhibitor Bortezomib Does Not Affect Radio- or Chemosensitivity. Transl Oncol. 2019; 12: 417-25.

41. Tomaic V. Functional Roles of E6 and E7 Oncoproteins in HPV-Induced Malionancies at Diverse Anatomical Sites. Cancers (Basel). 2016: 8

42. Schroder K, Tschopp J. The inflammasomes. Cell. 2010; 140: 821-32.

43. Lanfranca MP, Mostafa HH, Davido DJ. HSV-1 ICP0: An E3 Ubiquitin Ligase That Counteracts Host Intrinsic and Innate Immunity. Cells. 2014; 3: 438-54

44. Merkl PE, Knipe DM. Role for a Filamentous Nuclear Assembly of IFI16, DNA, and Host Factors in Restriction of Herpesviral Infection. MBio. 2019; 10.

45. Lo Cigno I, De Andrea M, Borgogna C, Albertini S, Landini MM, Peretti A, et al. The Nuclear DNA Sensor IFI16 Acts as a Restriction Factor for Human Papillomavirus Replication through Epigenetic Modifications of the Viral Promoters. J Virol. 2015; 89: 7506-20.

46. Singh VV, Kerur N, Bottero V, Dutta S, Chakraborty S, Ansari MA, et al. Kaposi's sarcoma-associated herpesvirus latency in endothelial and B cells activates gamma interferon-inducible protein 16-mediated inflammasomes. J Virol. 2013; 87: 4417-31.

47. Li T, Chen J, Cristea IM. Human cytomegalovirus tegument protein pUL83 inhibits IFI16-mediated DNA sensing for immune evasion. Cell Host Microbe. 2013; 14: 591-9.

48. Swatek KN, Usher JL, Kueck AF, Gladkova C, Mevissen TET, Pruneda JN, et al. Insights into ubiquitin chain architecture using Ub-clipping. Nature. 2019; 572: 533-7.
49. Gagnon D, Archambault J. A high-throughput cellular assay to quantify the p53-degradation activity of E6 from different human papillomavirus types. Methods Mol Biol. 2015; 1249: 111-20.

50. Tommasino M, Accardi R, Caldeira S, Dong W, Malanchi I, Smet A, et al. The role of TP53 in Cervical carcinogenesis. Hum Mutat. 2003; 21: 307-12.

51. Masuda Y, Saeki Y, Arai N, Kawai H, Kukimoto I, Tanaka K, et al. Stepwise multi-polyubiquitantion of p53 by the E6AP-E6 ubiquitin ligase complex. J Biol Chem. 2019.

52. White EA, Munger K, Howley PM. High-Risk Human Papillomavirus E7 Proteins Target PTPN14 for Degradation. MBio. 2016; 7.

53. Karim R, Tummers B, Meyers C, Biryukov JL, Alam S, Backendorf C, et al. Human papillomavirus (HPV) upregulates the cellular deubiquitinase UCHL1 to suppress the keratinocyte's innate immune response. PLoS Pathog. 2013; 9: e1003384.

54. Dickson C, Fletcher AJ, Vaysburd M, Yang JC, Mallery DL, Zeng J, et al. Intracellular antibody signalling is regulated by phosphorylation of the $\mathrm{FC}_{\mathrm{C}}$ receptor TRIM21. Elife. 2018; 7.

55. Jiang Y, Zhu Y, Liu ZJ, Ouyang S. The emerging roles of the DDX41 protein in immunity and diseases. Protein Cell. 2017; 8: 83-9.

56. Zhang Z, Bao M, Lu N, Weng L, Yuan B, Liu YJ. The E3 ubiquitin ligase TRIM21 negatively regulates the innate immune response to intracellular double-stranded DNA. Nat Immunol. 2013; 14: 172-8.

57. Li D, Wu R, Guo W, Xie L, Qiao Z, Chen S, et al. STING-Mediated IFI16 Degradation Negatively Controls Type I Interferon Production. Cell Rep. 2019; 29: $1249-60$ e4.

58. Natarajan V. Mind the Gap between the Endothelium and E3 Ubiquitin Ligase: TRIM21 is a Viable Therapeutic Target in Sepsis-Induced Endothelial Dysfunction. Am J Respir Cell Mol Biol. 2019.

59. Bottermann M, James LC. Intracellular Antiviral Immunity. Adv Virus Res. 2018; 100: 309-54. 